\title{
CLINICAL SCIENCE RESEARCH
}

Frederick L. Grover, MD A. Laurie Shroyer, $\mathrm{PhD}^{\mathrm{b}}$
Clinical science research incorporates the fields of clinical investigation and health services research. With a focus on the use of either human specimens or subjects, clinical investigation research projects translate knowledge gained from basic science research based on animal models for disease. The goal of clinical investigation is to develop new prevention, intervention, and therapeutic approaches to improve patient clinical outcomes. In contrast, health services research focuses on the improvement of the efficacy, costeffectiveness, and outcomes of care. Health services research projects examine options to improve the health care delivery system, organization, financing, and reimbursement mechanisms in place today. The purpose of this article is to review common terminology and methodologic approaches that are used in clinical science research. The process of designing a research project is reviewed. Beginning with the development of a research question and hypothesis, the steps for successful completion of the project are discussed. Different study design approaches are presented with their respective strengths and weaknesses. The challenges associated with conducting a clinical research study are discussed, including the development of an appropriate sampling strategy, the designing of data collection, instruments, and the assurance of study data integrity. Possible threats to study validity and generalizability are assessed.

One the major advantages of clinical research is that it offers an opportunity to study clinical questions in the clinical setting without the expenses of a basic research laboratory and basic science technology. Thus important clinical questions related to patient care, new technology assessment, clinical practice management, health care administration, or health policy may be addressed. (J Thorac Cardiovasc Surg 2000;119:S11-21)
I the field of clinical science, there are several categories of research approaches that may be appropriate to consider for either a clinical investigation or a health services research project. Traditionally, research in the field of medicine has been dedicated to basic science with a mechanistic focus that uses animal models for disease. More recently, the fields of clinical investigation and health services research have emerged as dis-

From the Division of Cardiothoracic Surgery, Department of Surgery, ${ }^{a}$ and the Division of Internal Medicine, Department of Medicine, ${ }^{\mathrm{b}}$ University of Colorado Health Sciences Center, Denver Department of Veterans Affairs Medical Center, Denver, Colo.

Supported in part by the Department of Veterans Affairs Health Services and Research grant \#IHY 99214-1 (A.L.S.).

Address for reprints: Laurie Shroyer, MD, Denver Department of Veterans Affairs Medical Center, 1055 Clermont St (112R), Denver, CO 80220 (E-mail: Laurie.Shroyer@ Med.va.gov).

12/0/104720

doi:10.1067/mtc. 2000.104720 tinct options toward the long-term goal of the improvement of the quality of patient care.

Clinical investigation research is the discipline by which physicians and other professionals translate knowledge gained in basic science to develop both preventive interventions for diseases and to improve the outcomes of diseases in patients. With either human subjects or specimens, this discipline focuses on the development of new or refined methods of diagnosis and treatment for patients. ${ }^{1}$

The discipline of health services research focuses on efficacy, cost-effectiveness, and outcomes of diagnostic, preventive, interventional, and therapeutic approaches for patients. Health services research projects evaluate the delivery, organization, and financing mechanisms in health care. ${ }^{2}$ Common study endpoints for a health services research study include traditional clinical outcomes (such as mortality or morbidity rates), health-related quality of life, patient satisfaction, costs of care, or access to care measures. 
From a study design viewpoint, both the clinical investigation and health services research categories for clinical science research design approaches include (but are not limited to) (1) traditional scientific experiments, (2) quasi-experimental approaches, and (3) observational clinical outcome studies. These clinical science research studies can be designed as either retrospective reviews or prospective studies. The sampling method selected may be randomized or nonrandomized. Moreover, the study design may be either blinded or double-blinded. Finally, either a single-institutional or multi-institutional approach may be taken. In summary, the methodologic approaches used for clinical science research projects are nearly as diverse as the research questions addressed. Thus the purpose of this article is to review several common terminology and methodologic approaches used as well as to raise the awareness of clinicians in the pursuit of these types of approaches for important clinical science research questions.

One of the advantages of clinical science research is that it offers an opportunity to study clinical questions in an organized fashion in "real life" situations. It does not require the expenses of a basic research laboratory and basic science technology, and it can be performed as part of academic or private practice. In addition, clinical science research cannot be criticized because of species differences, because it only relates to human subjects. It can be performed relatively economically, and it can be very practical and answer important everyday clinical questions related to patient care, practice management, or health policy.

\section{Overview of the research process}

The framework for scientific inquiry generally involves the evolution from theory to reality. Although research projects may either be deductive (hypothesis testing) or inductive (hypothesis generating) in nature, clinical science research appears to be predominantly hypothesis-driven research to date. To appropriately design and implement a clinical science research project, therefore, there are several key components that must be included.

Fig 1 shows the research project design process, beginning with the development of a research question and the corresponding hypothesis, the establishment of a supporting conceptual framework for the hypothesis, and testing the hypothesis. ${ }^{3}$ To test a hypothesis, the variables that will be measured must be identified and clearly described, the data capture plan must be outlined, and the data measurement integrity must be verified. From a perspective of determining measurement integrity, a strategy to determine the validity (how accurately the instruments used measure the constructs that you are attempting to measure) and the reliability (how reproducibly and stably your measurement instrument performs) need to be established.

Next, options for possible study design approaches need to be evaluated. In context of other project limitations (such as feasibility, ethical considerations, or budget constraints), the advantages and disadvantages of each alternative study design approach need to be assessed. At a minimum, the study method chosen should adequately address the primary research question of interest.

After the study design approach is selected, then the data collection strategy (including a plan for the data collection instrument design and testing) needs to be developed. Correspondingly, both database programming and data quality-control efforts need to be outlined. Within this study design context, the analysis plan and corresponding sample size calculations need to be coordinated.

From many years of experience, the key to successful project planning is to assure the internal consistency among all aspects of the research project plan. The study hypothesis, conceptual model linkages to existing theory and literature, study design approach selected, analysis plan, and sample size calculations should synchronize to clearly address the research question posed. For example, the sample size approach used should be based generally on the analytic approach planned. Any inconsistencies within the research project proposal should be clearly justified.

Often, a project budget must be developed. This budget should be realistic, and potential sources of funding must be identified. Depending on the possible funding agency, a letter of intent may have to be submitted, followed by a formal grant proposal. However, the specific format and content requirements of each funding agency grants program may differ.

An administrative structure for the planned study should also be established (ie, Who are the principal investigator, co-investigator, consultants, and collaborators? Who will participate in the study? Who will administer the study on a day-to-day basis? Who will collect the data and perform the planned analysis?) For a clinical science project, ideally a multidisciplinary team of clinicians, health services researchers, statisticians, and other study support personnel (such as database programmers, computer specialists, and others) should be assembled, based on the project's unique needs. Thus a study implementation plan that carefully identifies the roles and responsibilities of the study team members is well advised. 


\section{Image available in print version only}

Fig 1. Research project design process. The first step is to develop a research question and a corresponding hypothesis, then to create the supporting conceptual framework for that hypothesis, and to provide the means by which to test the hypothesis. The research method must be chosen; the population and sampling strategy must be identified; the event must be observed; and the data must be captured, processed, and analyzed. (Adapted from Babbie E. The practice of social research. 4th edition. Belmont [CA]: Wadsworth; 1986. Reprinted with permission of Wadsworth, a division of Thomson Learning. Fax 800 730-2215.)

For most clinical science studies, the proposed study should be reviewed and approved by the local Institutional Review Board, according to their standards. Where appropriate, informed consent forms may have to be obtained from the patient before entry into the study. Additional study implementation concerns relate to maintaining data confidentiality and security. Access to study data should be limited to key personnel where justified. Security precautions, such as password protection and encryption of patient-specific identifiers, should be planned. Confidentiality policies and agreements should be established to protect study participant privacy and to guard against unauthorized access or use to the data captured. From an ethical viewpoint, therefore, the research scientist is responsible for assuring the privacy, confidentiality, and security of the study data maintained.

Finally, the need for a scientific monitoring review committee should be assessed on the basis of the nature and scope of the clinical science study. This type of independent research project audit structure provides for a preliminary review of the study data at interim time points, to determine whether there is a need to stop the study prematurely. Possible reasons for early study closure include (but are not limited to) patient safety issues (such as unanticipated adverse reactions) or because of a superior effect being demonstrated early in 1 of the study arms. This type of external scientific review process, moreover, engenders public confidence and trust that clinical science projects are conducted appropriately.

Inherent to the design and implementation of a clinical science research project, therefore, is the need for overall consistency and synergy between these key study components. In any clinical science study, the success of the project will relate to the integration and successful completion of these critical study design components, as the building blocks in the project's foundation.

\section{Terminology}

In research, there are several basic concepts and definitions (both individual terms and the interrelation- 
ships between these concepts) that one should understand. ${ }^{3}$ The first concept is related to the scientific term hypothesis. A hypothesis is a formal and testable statement of relationships between dependent and independent variables. The dependent variable is the study outcome of interest. Clinical science research studies may evaluate clinical outcomes, cost-effectiveness of care, access to care issues, efficacy and effectiveness of new therapies, patient preferences, and patient choices. For example, the project's dependent variable may be mortality rates, morbidity rates, patient self-reported health-related quality of life scores (such as the SF-36 physical or mental summary component scores), patient satisfaction ratings, or patient utility scores. All of these examples of dependent variables relate to the possible outcomes that may be influenced by a health care intervention (such as a diagnostic test, treatment, operation) or episode of care (such as an emergency room visit). The independent variables are other variables of interest that may have an association with the dependent variable of study (including the intervention of study).

For research purposes, null hypotheses and alternative hypotheses are often identified. A null hypothesis normally states the lack of an association (or a random association) between the dependent and independent variables of study. An alternative hypothesis is usually the association of interest with an identified direction for the association, where appropriate. For example, a clinical science project may examine the impact of a surgical technique (such as off-pump cardiac bypass procedures as the independent variable of primary interest) on 30-day operative mortality rates (as the dependent variable studied). In this example, the null hypothesis might be stated, "There is no association between off-pump bypass procedure approach (as compared with a traditional surgical approach) and riskadjusted 30-day operative death rates." In contrast, the alternative hypothesis might be stated, "There is a relationship between the use of off-pump bypass procedures and increased risk-adjusted 30-day operative death rates." To appropriately address other patientspecific risk factors (as other independent variables that may also potentially influence outcomes), a risk-adjustment process with multivariate logistic regression modeling is often used. Thus the impact of off-bypass procedures may be examined on operative mortality rates, holding all other patient risk factors constant.

The second key concept to define is what is a fact. A fact is an observation that may be repeated by either the same observer or multiple observers to give the same result. For example, a relevant fact may be the cardiac surgery patient's age that may influence the patient's risk of adverse events.

The next concept is a law. A law is a generalization about a class of facts. Laws often are related to a wellestablished set of principles by summarizing patterns in scientific findings. For example, it has been well established in the cardiac surgery field that as patient age increases, the risk of adverse events increases also.

Theory, in contrast, is a systematic explanation of a set of facts and laws. For predictions of risk in cardiac surgery, Jones and colleagues ${ }^{4}$ have assimilated findings from multiple national databases to identify a set of consistent "core" risk factors that may influence risk-adjusted outcomes of care. These risk factors include acuity, previous heart surgery, age, ejection fraction, sex, number of diseased vessels, and the presence of left main disease. To be consistent with the theory in this field, therefore, new clinical research studies that were were developed to predict risk of death in cardiac surgery often incorporate these core variables.

Finally, there is the concept of a paradigm, which is a model or scheme that organizes our world view and simplifies our understanding of reality. For example, the paradigm of continuous quality improvement places a value on provider self-assessment and selfimprovement. In this context, risk-adjusted outcomes may be used to facilitate changes to improve quality of care. Reporting risk-adjusted outcomes is not an end unto itself but rather a means to an end. A primary goal of clinical science research, therefore, is to use hypothesis-driven research to document facts that may alter the laws and theories that are developed, which may subsequently redefine our paradigm of how to improve the quality of medical care in the future.

\section{Common study design approaches}

From a research methods viewpoint, both clinical investigation and health services research studies commonly use certain study design approaches. These approaches include (but are not limited to) (1) scientific experimental approaches, (2) quasi-experimental methods, and (3) observational clinical outcome studies. Although these methods are not unique to the field of clinical science, it is important to understand the strengths and weaknesses of each approach to answer clinical science research questions.

Fig 2 shows the scientific experimental study design, where subjects are randomized to either the intervention or control group..$^{5}$ In this approach, the outcomes and covariates are measured in both groups at baseline. After the administration of an experimental stimulus 


\section{image available in print version only}

Fig 2. Experimental approach. A scientific experimental design is shown where subjects are randomized to either an intervention or a controlled group, and the dependent variables and covariants, which are measured at baseline in either an experimental stimulant or a placebo (control group), are administered. The dependent variable is remeasured to assess the effect of experimental stimulus. (Adapted from Babbie E. The practice of social research. 4th edition. Belmont [CA]: Wadsworth; 1986. p. 184. Reprinted with permission of Wadsworth, a division of Thomson Learning. Fax 800 730-2215.)

(the intervention of interest), the outcomes are remeasured in both the experimental and control group. Given that clinical science studies are conducted on human subjects and specimens, it may not always be possible to control the experimental environment, the intervention, and all possible other factors that may influence the outcomes studied. This type of study is often called a randomized, controlled clinical trial. Although this study design represents the most rigorous approach possible, it may not always be feasible or possible to conduct this type of a research study, because randomization may not be feasible. Moreover, this type of experimental approach may not reflect reallife clinical situations.

A second preference is a variety of quasi-experimental approaches. If subjects are not randomized, then it may be possible to develop a set of comparison and intervention groups, which are similar, based on a matching process. Fig 3 shows the similarity of a quasiexperimental approach to a traditional experimental study design. Although not as rigorous as a randomized, controlled clinical trial, this type of approach may often be used to answer important clinical questions.

For both experimental and quasi-experimental studies, the allowance for crossovers from 1 arm of the study to another arm (as well as predefined criteria for study failures) need to be addressed. The usual approach, however, is to analyze the data conservatively, according to the intention to treat (ie, according to the original arm in which they were placed). An example of this might be an angioplasty versus coronary bypass intervention study, where a patient may have been originally assigned to angioplasty. Given changes in the patient's clinical condition (according to prespecified criteria), however, the patient is crossed-over to coronary artery bypass. This patient, however, would normally be analyzed as receiving an angioplasty rather than a bypass procedure.

If an intervention-based trial is not reasonable to pursue, then observational studies are used to explore associations between exposure to a given intervention (or episode of care) with an outcome of interest. For a given predefined patient population, possible study design approaches include case-control studies (where subjects are selected on the basis of the presence/ absence of the outcome of interest) or cohort studies (where subjects are selected on the basis of exposure or nonexposure to the intervention of interest) ${ }^{5}$ These traditional epidemiologic approaches to study design, however, are limited because they cannot be used to demonstrate causality.

For many observational studies, it may be possible to use secondary source data (data collected for purposes other than the research project) as an unobtrusive approach to answer key clinical science questions. Other possible observational study approaches include meta-analysis (a systematic technique for analyzing a set of studies), content analysis (a review of data to 


\section{Image available in print version only}

Fig 3. Quasi-experimental approach. In a quasi-experimental approach, the subjects are not randomized; instead, a comparison group is developed. The comparison group includes cases that are similar. Where appropriate, a matching process may be used. Although not as rigorous as a randomized, controlled clinical trial, this type of approach can be useful to answer important clinical questions. (Adapted from Babbie E. The practice of social research. 4th edition. Belmont [CA]: Wadsworth; 1986. p. 184. Reprinted with permission of Wadsworth, a division of Thomson Learning. Fax 800 730-2215.)

examine for patterns and to develop hypotheses about possible relationships between variables), and analysis of existing summary statistics (such as United States census data) to evaluate for trends or to make comparisons across groups. These different types of study approaches may be relatively inexpensive and timely and may perhaps facilitate the addressing of questions that may be normally beyond the data-capture abilities of an individual investigator.

Analysis of secondary source data may be used to find patterns and associations that might otherwise not be explored. However, there are possible disadvantages to these types of studies that use secondary source data. First, because the data was captured for purposes other than the research question of interest, not all of the key data elements that should ideally be present for analysis may actually be available. Second, the researcher is at the mercy of the agency (as well as agency politics) that controls the access to the data and is dependent on the completeness and quality of the data available. Finally, the conclusions drawn from the study findings need to be thoughtfully placed in context of any known assumptions (and possibly unknown factors) that may have influenced the study's results.

Studies may be designed as either cross-sectional (based on observation assessed at a single point in time) or longitudinal (based on observations made at multiple time points). Although the possible study design approaches to be used (and subtle permutations on these approaches) are beyond the scope of this article, these 3 approaches (experimental, quasi-experimental, and observational studies) are common strategies used in the field of clinical science.

\section{Threats to study validity}

Clinical science research presents many challenges. When organizing a study, one has to guard against threats to both external and internal validity. External validity relates to the generalizability of the study's population. For example, to what degree is the patient population enrolled or observed representative of the general population of patients with the disease of interest? Concerns of external validity may limit the application of a given study's conclusions to other populations or care settings.

Maximizing internal validity relates to minimizing systematic bias. Possible areas of concern where bias may commonly arise include (1) an enrollment bias (that the patients who are enrolled are different in some important way from those patients who did not enroll), (2) a follow-up bias (that the patients for whom followup data were procured are different in some important way than those patients who were lost to follow-up), or (3) a compliance bias (that the patients who complied with the intervention/treatment regimen are different in some important way than those patients who did not). 


\section{Annual Use Of IMA Conduit Summary}

\section{U.S. Data First Op CAB}

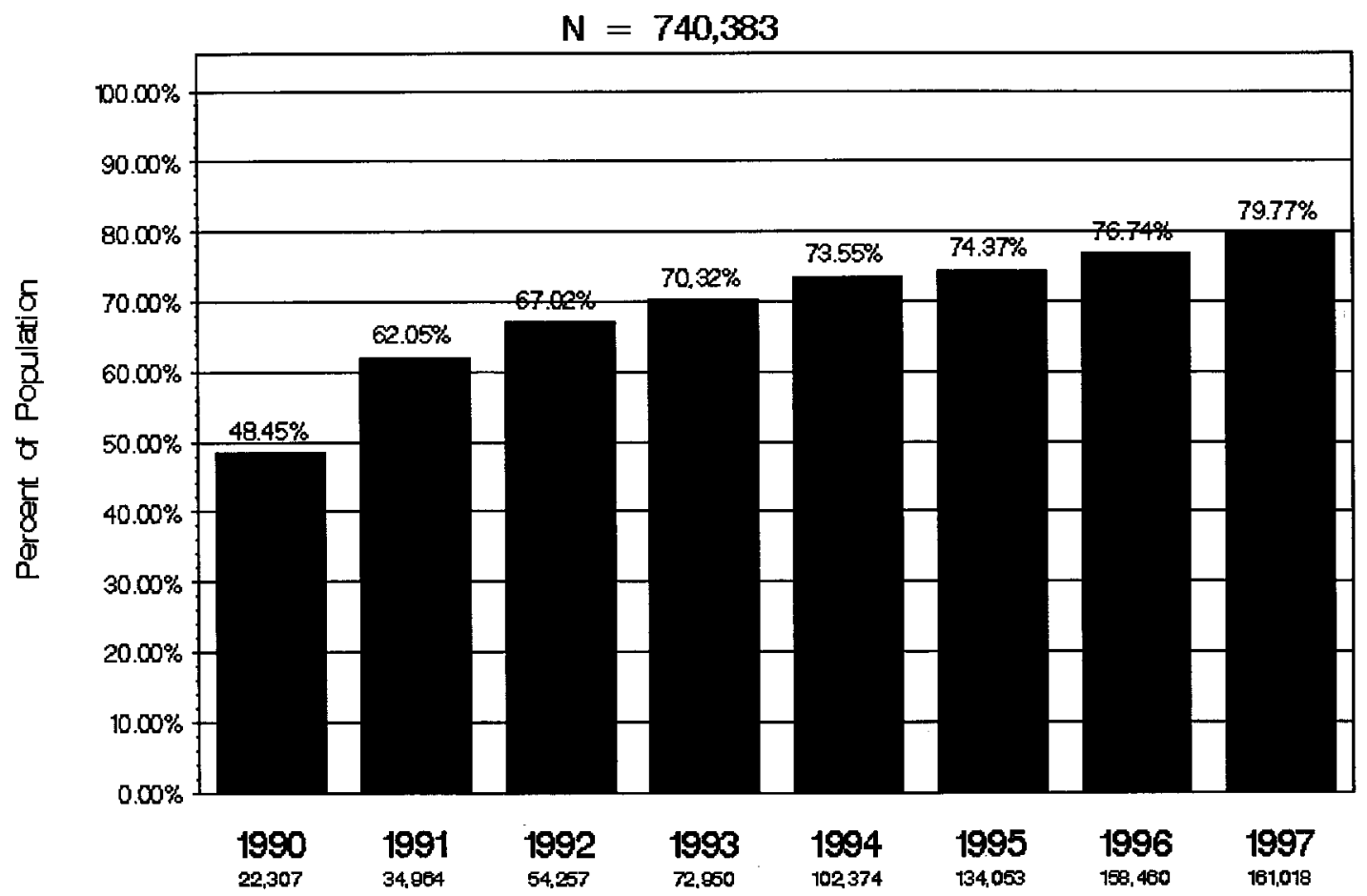

Fig 4. Incidence of the use of the internal mammary artery (IMA) in first-time CABG procedures in the STS database. Note that, from 1990 to 1997, there has been a marked increase in the use of at least 1 internal mammary artery in coronary bypass procedures, increasing from $48.45 \%$ to $79.77 \%$. Data such as this from observational databases can serve as benchmark comparisons for practicing clinicians. (From data analysis of The Society of Thoracic Surgeons National Adult Cardiac Surgery Database, The Eight Year, December 1998.)

Systematic bias may completely invalidate a study's findings.

External influences that may potentially bias the study's results should also be carefully considered. Where possible, these external influences should be measured as study covariates. Unmeasured influences represent possible confounding variables. Examples of possible external influences include contamination between the study groups, where the control and intervention arms may exchange information or compare treatment regimen results informally. Key issues may arise that are related to the knowledge of the identity of study and control group assignment. Thus a single-blinded approach (where either the subject or the researcher does not know the study arm assigned) or double-blinded approach (where neither the subject or the researcher knows the arm assignment) may be used to proactively prevent this type of problem from occurring.
Another issue of concern in clinical science research is that, during the course of a study, changes in clinical practice can take place that could affect the outcome of the study. An example of this would be technologic improvements that yield progressively better results during the course of the study, rendering the first part of the study irrelevant and/or outdated. Thus a timedependent association with the outcome of interest may be present.

\section{Sample size issues}

Ethically, it is important not to study too many subjects or too few subjects, because both of these approaches may be wasteful or detrimental (either directly to patients or to the future knowledge base in this field). Thus it is important to determine the sample size (an estimate of the number of subjects needed) as part of the study design process. 
An adequate sample size addresses a common human fallacy: to overgeneralize inappropriately on the basis of the results found for a few cases. Moreover, an adequate sample size minimizes the possibility of a type II ( $\beta$ ) statistical error. For reference, a type II error is a false negative (that is, the null hypothesis is actually false, but it has not been disproved appropriately). With a larger sample size (which correspondingly increases statistical power), however, a statistical difference might have been detected to disprove the study's null hypothesis.

As a direct result of sample size calculations, it may be self-evident that adequate accrual to the planned clinical project will require a multicenter study. Multicenter studies present additional study design and implementation challenges. The key factor for success of a multicenter study is the use of a uniform and standardized protocol. Common concerns in multicenter studies include possible interrater variability in data capture, institutional variability in protocol administration/adherence, the diverse levels of dedication to the study by the key participating investigators, the continued accrual of patients, and communication issues with the central study leadership team.

\section{Research funding opportunities}

Numerous funding options are available for clinical science studies. Although there are exceptions, however, most grant opportunities require that the principal investigator hold a doctoral-level degree.

Grant funding may be available through local, state, regional, or national opportunities. Commonly, several national organizations fund clinical science research projects. For individuals interested in research questions that are related to cardiothoracic research topics, these organizations may include the National Institutes of Health, the Agency for Health Care Policy and Research, the Department of Veterans Affairs (both Health Services Research and Cooperative Studies Programs and the Clinical Merit Review Programs), the Health Care Financing Agency, the American Heart Association, the American College of Surgeons, pharmaceutical and device industries, foundations (such as the Thoracic Surgery Foundation for Research and Education), and trauma organizations, as well as many other sources. The possibilities for funding should be investigated carefully to determine a match between the agency's funding goals and the planned objectives of the research project.

\section{Clinical science studies}

Several examples of clinical science research projects will be reviewed, beginning with single-center studies.
An example of a retrospective, observational study is entitled, "Single Lung Transplantation: Alternative Indications and Technique." 6 This article by Calhoon and colleagues hypothesized that a telescoping bronchial anastomosis without omental wrap was as efficacious as the use of a more complicated technique. This retrospective review of 22 patients (who had undergone 23 single lung transplantations) was performed at a single site. It had no control group, using a historic comparison based on the available literature. The results demonstrated that this technique was comparable to the previously published literature for mortality rate and rate of perioperative complications. This article's conclusion, therefore, was to use the more simple technique because the results were as good.

Another classic example of a retrospective observational study is the study by Loop and colleagues ${ }^{7}$ entitled, "Influence of the Internal Mammary Artery Graft on 10-year Survival and Other Cardiac Events." This single-center, retrospective cohort study compared 10year actuarial survival rates between 2 groups. The intervention group of interest involved the use of an internal mammary artery to the left anterior descending coronary artery in comparison with the group of patients with only saphenous vein graft procedures. Both groups of patients that were reviewed underwent operation during a comparable time period. The patient outcomes that were evaluated included the actuarial survival, incidence of myocardial infarction, rehospitalization, cardiac reoperation, and late cardiac events. The article's conclusion was that the internal mammary artery conduit was preferable when feasible, because the results appeared to be superior.

An alternative common type of single-center study published is a prospective, double-blinded, randomized controlled clinical trial. These studies are planned and submitted before the study to a local Institutional Review Board with a set protocol, sample size projection, and a plan for statistical analysis. One example is a study reported in 1977 entitled, "Prophylactic Antibiotics in the Treatment of Penetrating Chest Wounds: A Prospective Double Blind Study." 8 In this study the hypothesis was that antibiotics would reduce infection and sepsis in penetrating chest trauma. The study inclusion population was confined to those patients with penetrating chest trauma. Patients were randomized to 2 groups, those receiving antibiotics and those receiving a placebo. The outcomes measured were death, temperature, white blood cell count, culture data, evidence of wound sepsis, radiograph evidence for pneumonia or effusion, emphysema, and operative interventions. The conclusion of the study was that prophylactic antibiotics decreased pneumonia, 
the incidence of fever, emphysema, wound infection, operations, and length of hospital stay. This was a fairly simple study that had (as many single-center studies often have) a borderline sample size. Additionally, it is possible that a single-center bias may exist that is related to the other processes of care that may have influenced the outcomes of study.

The next example of a single-center prospective analysis by Fullerton and colleagues ${ }^{9}$ is entitled, "Effective Control of Pulmonary Vascular Resistance with Inhaled Nitric Oxide after Cardiac Operation." This study tested the hypothesis that nitric oxide would selectively decrease pulmonary vascular resistance in patients after cardiopulmonary bypass. Twenty patients received nitric oxide. Given that assessments of outcome were performed both before, during, and after nitric oxide administration, these patients served as their own controls. The outcome variables measured were pulmonary artery pressure, pulmonary vascular resistance, systemic vascular resistance, and arterial blood pressure. It was concluded that pulmonary vascular resistance was selectively decreased by nitric oxide in this group of patients.

These 3 clinical science research projects are therefore examples of relatively straightforward, simple studies that can be done by most clinicians at a single center to answer clinically important and pragmatic questions.

When a greater sample size is required and the potential biases of a single center need to be minimized, a multicenter study should be considered. In general, most multicenter studies are prospective, although it is, of course, possible to pool data from multiple institutions for retrospective analyses. Almost always, however, a more powerful and convincing study is a prospective randomized controlled clinical trial with pre-established criteria for patient inclusion, standardized protocol administration, and uniform outcome analysis.

Two examples of prospective multicenter scientific studies are the Coronary Artery Surgery Study (CASS) Study ${ }^{10}$ and the Veterans Affairs Aspirin Study that related to coronary bypass grafting patients. ${ }^{11} \mathrm{CASS}$ was a randomized trial of coronary artery bypass surgery that studied survival data as an outcome. It was multicentered with a prospective patient registry and a controlled clinical trial between coronary bypass and medical treatment in patients with Canadian Cardiovascular Society Class 1 and 2 angina. The CASS hypothesis was that the coronary artery bypass graft $(\mathrm{CABG})$ procedure would decrease death and ischemic events in patients with coronary artery disease as compared with medical treatment. The CASS registry included 16,626 patients. However, only 2099 patients were eligible for randomization, and of that number, only 780 patients were randomized. Of those patients assigned to medical therapy, $24 \%$ of the patients were crossed over to surgery but were analyzed as if they were treated medically. This is an example of the difficulties that are encountered when very complex multicenter perspective studies are designed with the best intentions, in that a very small proportion of eligible patients were actually randomized for study.

The findings were that there was no significant difference in mortality rates between groups. The conclusion was that excellent survival rates occurred in both groups, which supported delaying CABG until more symptoms developed. That conclusion may well be a real and relevant one, but critics could argue that a large number of patients were excluded, limiting the study's generalizability. Entrance criteria based only on class 1 and 2 symptom levels was so narrowly defined that it would be improbable that indeed there would be a survival benefit of 1 therapy over the other. Nevertheless, it probably did serve to answer questions in that select group of patients.

The next example is a study by Goldman and colleagues ${ }^{11}$ on the effects of aspirin when given beyond 1 year after operation in patients who have undergone CABG. This was a multicenter Veterans Affairs cooperative study in which the hypothesis was that aspirin would increase patency. It was prospective, doubleblinded in approach and compared groups receiving aspirin therapy with groups receiving placebo. The end points were angiography at 3 years. Conclusions were that aspirin probably does not improve patency when given beyond 1 year after CABG. This was a study that had a high percentage of follow-up with a gold standard for measurement of coronary patency (ie, coronary angiography at 3 years). Again, this answered a very specific, simple, pragmatic clinical question.

The next category of clinical studies is clinical outcomes of observational studies that fall within the health services research area. Examples of this type of study are related to analyses conducted with the use of pre-established databases. Databases used for this purpose include the Society of Thoracic Surgeons (STS) National Cardiac Surgery Database, the Department of Veterans Affairs Continuous Quality Improvement in Cardiac Surgery Program Database, the Northern New England Cardiovascular Database, the New York State Database, and single institution large databases, such as the Duke Database. Many of these well-established, large databases include data on patient risk characteristics, procedural details, and outcomes of care after surgical procedures. 
Descriptive analyses (to document patient characteristics and procedure usage) can be performed to understand variations in patterns of practice. For example, the STS database had approximately 450 participating member sites from across the United States that reported $1,074,689$ procedures in the period from 1990 to 1997. ${ }^{12}$ During this same time, the operative mortality rate for CABG procedures fell from $4.59 \%$ (1990) to $2.54 \%$ (1997). Annual use of the internal mammary artery for first-time CABG procedures increased from $48.45 \%$ (1990) to $79.77 \%$ (1997) (Fig 4). Moreover, the reported median postoperative length of hospital stay dropped for first operation, elective CABG procedures decreased from 8 days (1990) to 5 days (1997). With the use of statistical analysis techniques, riskadjusted outcomes of care and resource usage measures can be reported both in patient subpopulations and in aggregate. For example, the mean postoperative length of hospital stay reported by clinical groupings for riskadjusted operative death rates in the 1995 to 1996 STS database were (1) very low-risk grouping (0\%-2.5\% predicted risk), 8.4 days; (2) low-risk grouping (>2.5\%$5.0 \%$ predicted risk), 11.9 days; (3) intermediate-risk grouping (>5.0\%-10.0\% predicted risk), 12.8 days; (4) moderate-risk grouping ( $>10 \%-20 \%$ predicted risk), 14.8 days; (5) high-risk grouping (>20\%-30\% predicted risk), 16.1 days; and (6) very high-risk grouping (>30.0\% predicted risk), 17.2 days. Moreover, these STS summary data are publicly available on the web site (www.sts.org). Thus this data may be very useful for hospitals or surgical group practices to use as benchmarks and as historic reference information for research purposes.

Recently, a new mechanism to access the STS National Adult Cardiac Database has been made available through mechanisms listed on the STS web site. An open invitation to cardiac surgeons with an interest in health services research has been made to explore key research issues with the use of this database..$^{13}$ Thus requests for special research project analyses are now being accepted.

These databases can be analyzed for various outcome studies. Examples of the use of these databases include studies on the effect of the use of the internal mammary artery on 30-day mortality rates from the STS Database by Crawford and colleagues ${ }^{14}$ and by Grover and colleagues ${ }^{15}$ who used the Veterans Affairs Continuous Quality Improvement in Cardiac Surgery Program; the effect of gender on outcomes by Edwards and colleagues ${ }^{16}$ from the STS; the effects of volume on mortality rates from both the Veterans Affairs Continuous Quality Improvement in Cardiac Surgery
Program and STS databases, ${ }^{17,18}$ and an examination of variations in outcomes between institutions from the Northern New England database. ${ }^{19}$ In the Northern New England database efforts, a further exploration of these variations led to their ongoing quality improvement program in which best practices are shared between hospitals and surgeons to improve the quality of patient care.

Fundamentally, observational studies that use secondary source data (such as these databases) provides an opportunity to conduct studies that would not otherwise be feasible to the individual investigator. Primary clinical science research project constraints that use secondary source data, however, may be related to data integrity (both completeness and quality) and to the fact that the research questions must be limited to the data available.

In addition, there are various clinical science research studies that are conducted prospectively, such as the study by Shroyer and colleagues ${ }^{20}$ that was funded by the Department of Veterans Affairs entitled, "The Processes, Structures, and Outcomes of Care in Cardiac Surgery Study Protocol.” This study gathered a comprehensive set of patient risk factors, processes of care, structures of care, and outcomes for approximately 5000 patients who underwent a cardiac surgery procedure at 14 participating Veterans Affairs medical centers. An example of how a data set such as this can be used for a substudy analysis was recently published by Almassi and colleagues, ${ }^{21}$ whose objective was to investigate the incidence, the predictors, and the mortality rates associated with atrial fibrillation and its impact on length of stay. The Processes, Structures, and Outcomes of Care in Cardiac Surgery study population was 3855 patients who had undergone CABG-only procedures performed at 14 Veterans Affairs hospitals. The primary study outcome of atrial fibrillation was found in 1500 patients. The findings of Almassi and colleagues were that there were multiple predictors of atrial fibrillation and that this key complication increased overall patient mortality rates and length of hospital stay.

Recently, the Department of Veterans Affairs instituted a major clinical science research focus to monitor guideline compliance headed by John Feussner, MD, Chief of Research Services, and by John Demakis, MD, Head of Clinical Outcomes Studies. This national initiative is entitled, "Quality Enhancement Research Initiative: QUERI." A variety of different research endeavors, including many new research studies related to ischemic heart disease, are ongoing as a part of this new initiative. 
As evidenced by the literature reference used earlier, health services research studies are fertile ground for cardiothoracic surgical investigators because they measure pragmatic issues that involve quality of care, access to care, and cost of care, all of which are issues that are exceedingly important in the current health care environment. Also, one does not have to have advanced academic training in either molecular biology or genetics to successfully perform these studies. Health services research, however, requires a multidisciplinary approach. Thus clinicians who are partnering with research scientists (with expertise in clinical research approaches and outcomes analysis) and biostatisticians (for the more complex statistical analyses of large population databases) are extraordinarily important.

\section{Summary}

The examples given earlier serve to support the value of clinical science research, as research options available to both inexperienced and experienced investigators alike. As described, these diverse studies span the realm of the inexpensive, single-center, retrospective studies to rather expensive, multicenter, prospective studies. They are, however, studies that should have appeal to clinical cardiothoracic surgeons because the data are obtained from the clinical arena. Designing these studies requires a scientific discipline, dedicated planning time to develop hypotheses and methods, and collaborative efforts with clinical research specialists, health care researchers, and statisticians. In so doing, the understanding of cardiothoracic patient care can be expanded and the quality of future patient care can be improved.

\section{REFERENCES}

1. "Clinical Research Curriculum Award." National Institutes of Health. Nov. 19, 1999. http://www.grants.nih.gov/grants/guide/ rfa-od-00-002.html.

2. Shi L. Health services research methods. Albany (NY): Delmar; 1997. p. 15-6.

3. Babbie E. The practice of social research. 4th edition. Belmont (CA): Wadsworth; 1986.

4. Jones RH, Hannan EL, Hammermeister KE, DeLong ER, O'Connor GT, Luepker RV, et al, for the Working Group Panel on Cooperative CABG Database Project. Identification of preoperative variables needed for risk adjustment of short-term mortality after coronary artery bypass graft surgery. J Am Coll Cardiol 1996;28:1478-87.

5. Hulley SB, Cummings SR. Designing clinical research: an epidemiologic approach. Baltimore (MD): Williams and Wilkins; 1988. p. 63-86.
6. Calhoon JH, Grover FL, Gibbons WJ, Bryan CL, Levine SM, Bailey SR, et al. Single lung transplantation: alternative indications and technique. J Thorac Cardiovasc Surg 1991;101:816-25.

7. Loop FD, Lytle BW, Cosgrove DM, Stewart RW, Goormastic M, Williams GW, et al. Influence of the internal-mammary-artery graft on 10-year survival and other cardiac events. N Engl J Med 1986;314:1-6.

8. Grover FL, Richardson JD, Fewel JG, Arom KV, Webb GE, Trinkle JK. Prophylactic antibiotics in the treatment of penetrating chest wounds: a prospective double-blind study. J Thorac Cardiovasc Surg 1977;74:528-36.

9. Fullerton DA, Jones SD, Jaggers J, Piedalue F, Grover FL, McIntyre RC Jr. Effective control of pulmonary vascular resistance with inhaled nitric oxide after cardiac operation. J Thorac Cardiovasc Surg 1996;111:753-63.

10. Coronary artery surgery study (CASS). A randomized trial of coronary artery bypass surgery: survival data. Circulation 1983; 68:939-50.

11. Goldman S, Copeland J, Moritz T, Henderson W, Zadina K, Ovitt $\mathrm{T}$, et al. Long-term graft patency (3 years) after coronary artery surgery: effects of aspirin: results of a VA Cooperative study. Circulation 1994;89:1138-43.

12. Society of Thoracic Surgeons National Database. Society of Thoracic Surgeons. http://www.sts.org/doc/2986.

13. National Database Access and Publication Subcommittee Committee Home Page. Society of Thoracic Surgeons. http://www.sts.org/committee/sts/394.

14. Crawford FA Jr, Anderson RP, Clark RE, Grover FL, Kouchoukos NT, Waldhausen JA, et al. Volume requirements for cardiac surgery credentialing: a critical examination: the Ad Hoc Committee on Cardiac Surgery Credentialing of The Society of Thoracic Surgeons. Ann Thorac Surg 1996;61:12-6.

15. Grover FL, Johnson RR, Marshall G, Hammermeister KE. Impact of mammary grafts on coronary bypass operative mortality and morbidity: Department of Veterans Affairs Cardiac Surgeons. Ann Thorac Surg 1994;57:559-69.

16. Edwards FH, Clark RE, Schwartz M. Impact of internal mammary artery conduits on operative mortality in coronary revascularization. Ann Thorac Surg 1994;57:27-32.

17. Shroyer AL, Marshall G, Warner BA, Johnson RR, Guo W, Grover FL, et al. No continuous relationship between Veterans Affairs hospital coronary artery bypass grafting surgical volume and operative mortality. Ann Thorac Surg 1996;61:17-20.

18. Edwards FH, Carey JS, Grover FL, Bero JW, Hartz RS. Impact of gender on coronary bypass operative mortality. Ann Thorac Surg 1998;66:125-31.

19. O'Connor GT, Plume SK, Olmstead EM, Morton JR, Maloney CT, Nugent WC, et al. A regional intervention to improve the hospital mortality associated with coronary artery bypass graft surgery. JAMA 1996;275:841-6.

20. Shroyer AL, London MJ, Villanueva CB, Sethi GK, Marshall G, Moritz TE, et al. The processes, structures, and outcomes of care in cardiac surgery study protocol. Med Care 1995;33(suppl): OS17-25.

21. Almassi GH, Schowalter T, Nicolosi AC, Aggarwal A, Moritz TE, Henderson WG, et al. Atrial fibrillation after cardiac surgery: a major morbid event? Ann Surg 1997;226:501-13. 